\title{
DISTINCT ELEMENT ANALYSIS OF DRY ROCK AVALANCHES
}

\author{
By Yoshihiko UCHIDA* and Motohiko HAKUNO**
}

\begin{abstract}
The mechanical behavior of a particle assembly is described based on Cundall's Distinct Element Method (DEM) which uses an explicit numerical scheme. The DEM is a numerical technique specifically designed to solve problems where continuity cannot be ensured throughout the analysis. We used this method to study the flow mechanism of dry rock avalanches which frequently take place following strong earthquakes. On the basis of the results obtained from a numerical simulation, we conclude 1) that the largest pieces of rock float up to the surface of the flow and 2) that they become concentrated at the front of the flow.

Keywords : dry rock avalanche, Distinct Element Method (DEM), Discrete Element Method (DEM)
\end{abstract}

\section{INTRODUCTION}

Methods for fracture analysis are divisable into two main categories:

a) those that deal with the medium as a continuous body

b) those that deal with the medium as a discontinuous body.

Examples of the first category are the normal Finite Element Method and Boundary Element Method. These can be applied to problems in which the medium is regarded as continuous even after fracture, but difficulties arise if the elements of the medium become separated after fracture. In the second category is the Distinct Element Method which can be used both before and after fracture.

The DEM used in the study reported here is a method for analyzing granular material, in which the medium is dealt with as an assembly of particles. For example, the ground is regarded as being composed of distinct elements (blocks) of soils and rocks. Internal stresses are transmitted through contact points, and an equation of motion is constructed for every distinct element, which allows observation of the movements of all the elements, one by one, in the time domain.

The object of the analysis was the rock avalanche; the downslope flow of soils, rocks, pebbles, pumice, etc. which has little water content. Rock avalanches that developed from slope failures have caused numerous disasters after earthquakes and volcanic eruptions. Because insitu experiments on this phenomenon tend to be expensive and dangerous, laboratory experiments are preferrred; but, there is the problem of duplicating the conditions of the actual phenomenon. We therefore devised an analysis of rock avalanches based on the DEM. Only a very few applications of this method have been made to rock avalanches. This is because of the enormous memory and large amount of computation time needed for

* Member of JSCE, Central Japan Railway Company (Nakamura-ku 1-1-4 Nagoya-city, Nagoya)

** Member of JSCE, Earthquake Research Institute, University of Tokyo (Yayoi 1-1-1, Bunkyo-ku, Tokyo) 
electronic computation. But, with progressive improvements in computers these requirements are being reduced year-by-year.

We undertook the study to confirm whether dry rock pieces flow down a slope like a liquid and whether large blocks float up and become concentrated at the front of a flow.

\section{PAST STUDIES OF THE DISTINCT ELEMENT METHOD (DEM)}

The DEM is a method for analyzing granular material. A number of analytical methods which deal with a granular medium as an assembly of particles have been proposed : the statistical mehtod of Mogamil), and the modified Satake method ${ }^{2)}$, among others. As a simulation method, the Discrete Block Method (DBM) proposed by Cundall ${ }^{3)}$ has been pivotal; it was the beginning stage for the present Distinct Element Method. The DBM was new in that the dynamic behavior of composite distinct blocks could be analyzed numerically in such a way as to satisfy the equations of motion for every element and to allow the transmission of forces between elements to follow the action-reaction law. In Japan, Hakuno and Hirao $0^{4)}$ dealt with the problem of a randomly packed spherical particles subjected to static shear. Kiyama and Fujimura $^{5)}$ analyzed ground subsidence produced by tunnel excavation and the gravity flow of rocky granular material in a silo using Cundall's method for equi-diameter cylindrical elements. Kawasaki and $\mathrm{Kiyama}^{6}$ ) made a DEM analysis using ellipsoidal elements for the medium with irregular pores that could not be modelled by cylindrical elements.

Uemura and Hakuno ${ }^{7)}$ increased the number of elements used from hundreds to several thousands and analyzed such problems as slope failure, behavior of ground during pile driving, vibrational response of a structure's foundation, and vibrational consolidation of ground.

Casaverde, Iwashita, Tarumi and Hakuno ${ }^{8)}$ analyzed slope failure, and Hakuno and Tarumi ${ }^{9)}$ analyzed the state in which interstitial pores are saturated (which had rarely been treated). The latter analysis simulated liquefied sand during an earthquake by taking into consideration the water pressure in a two-dimensional granular material.

Iwashita and Hakuno ${ }^{10)}$ expressed the effect of the material bonding elements by introducing pore springs and analyzed a variety of continuous to discontinuous grounds using the DEM exclusively. The cases dealt with were dynamic failures for two or three-dimensional cliffs.

Meguro and Hakuno ${ }^{11)}$ introduced multi-layer models to take the place of the homogeneous models that had been used in prior models. Thus it was possible to apply the DEM to multi-layer problems and reinforced concrete problems, and they were able to analyze large deformation of the surface of multi-layer ground owing to soil liquefaction and the failure of a concrete member.

\section{DISTINCT ELEMENT METHOD}

\section{(1) Outline of the Distinct Element Method (DEM)}

The DEM is a method for analyzing granular material. In a two-dimensional case the following equations of motion (1) and (2) are derived :

$$
m_{i} d^{2} V / d t^{2}+C_{i} d V / d t+F_{i}=0 \cdots
$$$$
I_{i} d^{2} \psi / d t^{2}+D_{i} d \psi / d t+M_{i}=0
$$

where $F_{i}:$ resultant force acting on the $i$-th particle, $M_{i}$ : resultant moment acting on the $i$-th particle, $C_{i}$ and $D_{i}$ : respective damping coefficients for to translation and rotation, which are specified as caused by the internal friction and air resistance on particles, $V_{i}:$ displacement vector, $\phi_{i}$ : rotation angle, $m_{i}$ : mass of the $i$-th particle, $I_{i}$ : moment of inertia of the $i$-th particle

In the preceding equations the resultant forces and moments are derived from the deformations of two springs inserted either normally or tangentially between the relevant particles (Fig. 1). These springs naturally have such non-linear characteristics as loss of resistance under tension in the normal direction or 


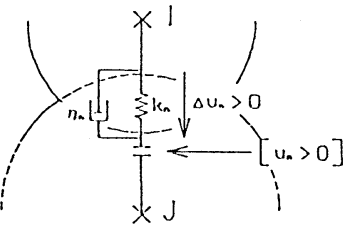

Normal direction

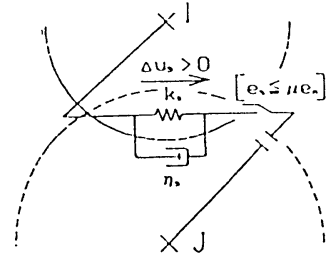

Transverse direction

Fig. 1 Mechanical model at contact point.

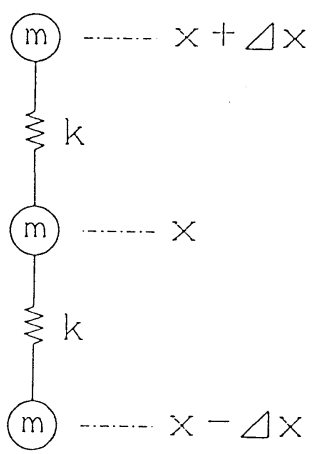

Fig. 2 Mass spring for the one-dimensional model.

slip over a limited friction force in the tangential direction. Therefore, the only unknowns in (1) and (2) are $v_{i}$ and $\psi_{i}$, which are obtained by converting the relations to finite difference equations and by integrating them in respect to time in the forward direction.

\section{( 2 ) Determination of parameters}

In the DEM, the stiffness or damping coefficients in the normal direction are not equal to the same coefficients of the real material; they are determined by taking into account physical considerations.

a) Determination of the stiffness coefficient

Assume the model shown in Fig. 2. Let the element mass be $m$ and the stiffness be $K$; the equation of motion is

$$
m d^{2} y / d t^{2}=K\left(y_{x+\Delta x}-y_{x}\right)-K\left(y_{x}-y_{x-\Delta x}\right)
$$

But, when the wave velocity is $C$, the wave equation is

$$
\partial^{2} y / d t^{2}=C^{2} \partial^{2} y / d x^{2}
$$

From the equation of vibration (3) we get

$$
m d^{2} y / d t^{2}=K\left(y_{x+\Delta x}-2 y_{x}+y_{x-\Delta x}\right)
$$

or

$$
d^{2} y / d t^{2}=K / m\left(y_{x+\Delta x}-2 y_{x}+y_{x-\Delta x}\right)
$$

where the distance between the elements is $\Delta x$ and the time step is $\Delta t$. Conversion of the wave equation to a difference equation, gives

$$
\partial^{2} y / \partial t^{2}=C^{2} / \Delta x^{2}\left(y_{x+\Delta x}-2 y_{x}+y_{x-\Delta x}\right)
$$

From equations (6) and (7) the following relation is derived approximately

$$
K=m c^{2} / \Delta x^{2}
$$

The stiffness coefficient can be determined from the above relation; but, it must be noted that this coefficient has been derived from a one-dimensional wave equation, equidiameter particles having been assumed, which is substantially different from the actual conditions. In a simulation this coefficient therefore may be used only as an approximate value, and $K$ must be modified; i. e., by measuring the velocity of the wave generated by an impulse given on the surface point of the model. This also applies to the coefficient in the tangential direction. We were satisfied if the assumed velocity could be reproduced approximately.

b) Determination of $\Delta t$

Analytical results are greatly effected by the time step $\Delta t$ because in the DEM analysis time-domain integration is conducted numerically. If the $\Delta t$ value is improper, various difficulties arise. When a wave passes through an element and is transmitted to the next element during one time step, the analysis may become unstable. This is because the wave is transmitted before the reaction from the contact element is derived, as the DEM is a method to transmit forces or waves through contact points between elements. Therefore, the condition that $\Delta t$ has to satisfy is 


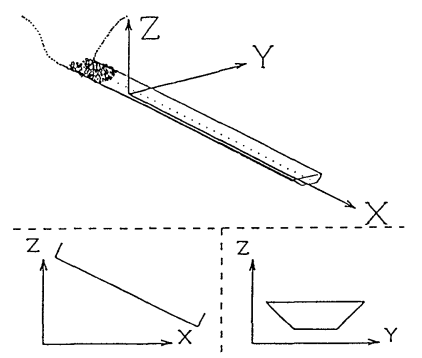

Fig. 3 Idealization of a rock avalanche.
Table 1 Assembly element data.

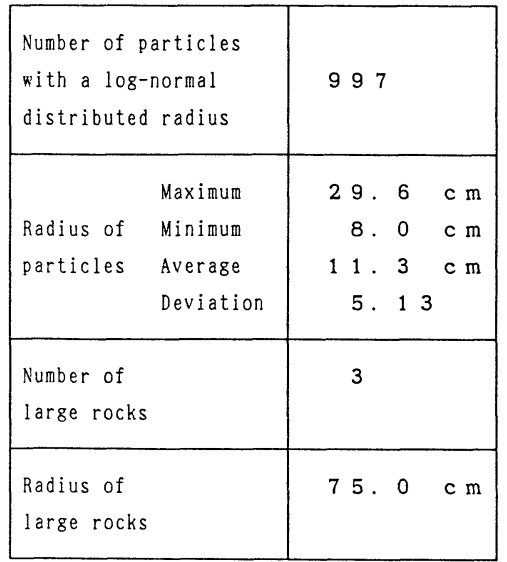

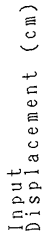

$$
\Delta t<\Delta x_{\min } / V
$$

where $V$ is the wave velocity. This condition implies that the distance that the wave is propagated in $\Delta t$ must be smaller than the minimum central distance between adjacent elements.

c) Modelling the rock avalanche

A very important point in DEM analysis is to model the medium in a realistic manner. Most natural phenomena are by nature three dimensional, and a rock avalanche is no exception. The channel through which the rock avalanche flows downward is both vertically uneven and horizontally winding. Although as an experiment is desirable to conduct a three-dimensional. DEM analysis, we made it in only two dimensions because of the limitation of computer time.

We reduced a three-dimensional rock avalanche to a two-dimensional one using the following two models

: The first is a longitudinal model which corresponds to the $x-z$ section in Fig. 3 , as the direction of the flow is in the $x$ direction. In this model the assembly of rock pieces (constitutive elements) was placed in the upper part of the slope, and its movement was analyzed solely under the effect of gravity. The second model is transverse one which crresponds to the $y$ - $z$ section in Fig. 3. In this analysis the longitudinal unevenness of the channel through which the rock avalanche flows was expressed by vertically shaking the vessel that contained the assembly. The results of both analyses could be examined separately, but combining them we could investigate the behavior of a rock avalanche in three-dimensional space.

\section{ANALYSIS OF THE FLOATING UP OF LARGE BOULDERS}

\section{(1) Explanation of the analysis model}

The model used was a cross-sectional one perpendicular to the flow direction of the rock avalanche. The channel through which the avalanche flows fluctuates laterally as well as vertically. In this analysis the vessel in which the particles are contained is shaken vertically as well as laterally to reproduce the effect of the change of the channel, and the behavior of the particles in the vessel is observed.

A trapezoidal vessel with a lower segment of 10 meters wide was prepared to contain the rock pieces 


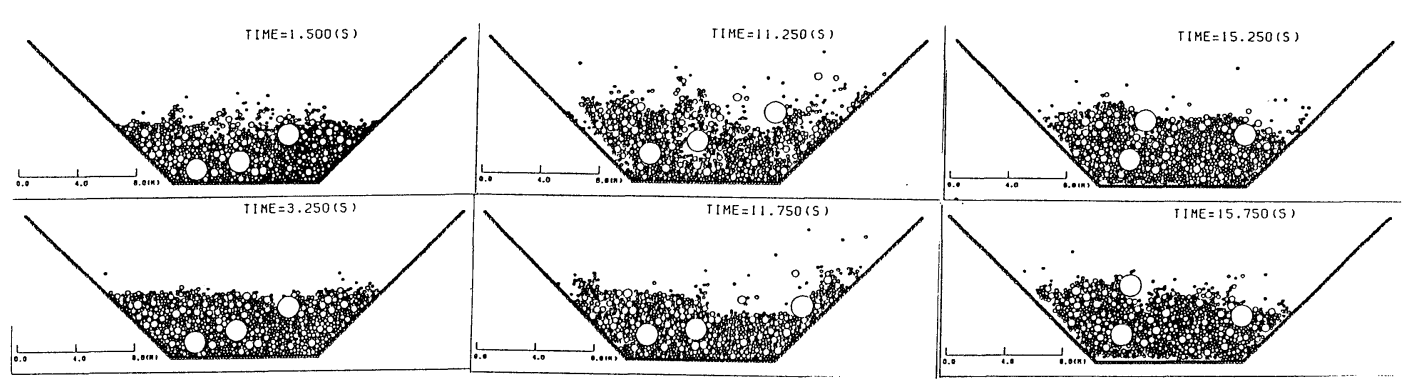

Fig. 6 Floating process of large rock produced by shaking of the cross section of the dry avalanche.

(wall elements). The radii of the wall elements were $10 \mathrm{~cm}$. Circular elements (radii $29.6 \mathrm{~cm}$ maximum, $8.0 \mathrm{~cm}$ minimum; mean $11.3 \mathrm{~cm}$ and variance 5.13 [Table 1]) were placed in the vessel (Fig. 4). The number of elements was 997. To observe the behavior of large boulders three elements each with a radius of $75 \mathrm{~cm}$, were mixed with the elements.

The behavior of the particles contained in the vessel was observed while the vessel was shaken vertically and laterally in a sinusoidal manner. The input sinusoidal motion is shown in Fig. 5, in which the vertical and lateral directions are denoted by the $z$ and $x$ axes. During the initial 8.5 seconds, the vessel was shaken only vertically with a maximum acceleration of $2 \mathrm{G}\left(\mathrm{G}=9.8 \mathrm{~m} / \mathrm{s}^{2}\right)$ and a period of 0.5 seconds. After 8.5 seconds, the maximum acceleration on the $z$-axis was increased to $4 \mathrm{G}$ and an acceleration of equal amplitude and period was given on the $x$-axis with a phase shift of

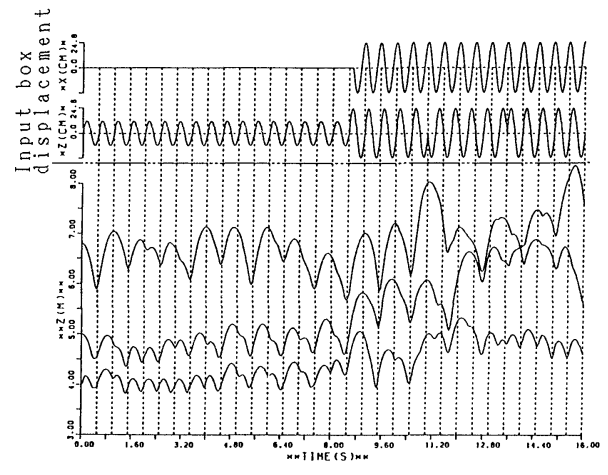

Fig. 7 Time histories of the positions of large rocks.

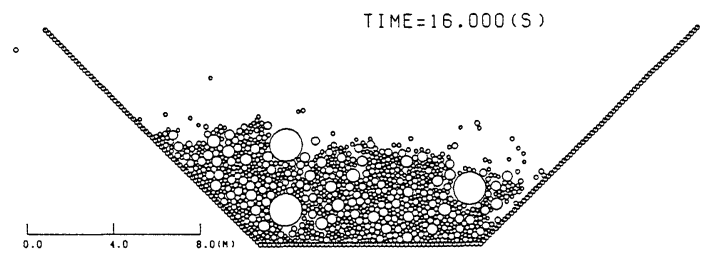

Fig. 8 Particle distribution at 16.0 sec of shaking. $1 / 4$ period.

\section{(2) Results}

The analytical results are given in Fig. 6. The time histories of the absolute coordinates of the three large boulders (elements with radii of $75 \mathrm{~cm}$ ) in the vertical direction $(z$-axis) are given in Fig. 7 . The lowest boulder was designated (1), the middle one (2) and the highest one (3) in the starting state in (Fig. 4).

The large boulder ( 3 ) appeared on the surface about 3 seconds after the start of shaking (Fig. 6). Fig. 7 shows that, the absolute coordinate of boulder (3) had not changed much from its initial position; therefore, the appearance of the boulder on the surface was the result of the relative motion to the surrounding particles.

After 8.5 seconds the upward movement of the large boulder (2) is remarkable. In view of its relative position in Fig. 7, it apparently moved after 12 seconds, and by 14 seconds was almost equal to the level of boulder ( 3 ) which had been highest of the three up to then. Boulder was unambiguously on the surface at 14.5 seconds and was the highest of the three boulders. Its upward motion also is apparent in Fig. 7, movement being especially remarkable after 8.5 seconds when the input acceleration was increased. Boulder ( 3 ) showed no tendency to subside among the particles after it once appeared on the surface at about 4.0 seconds, and it was still on the surface at 11.5 and 11.75 seconds. The final distribution of the 


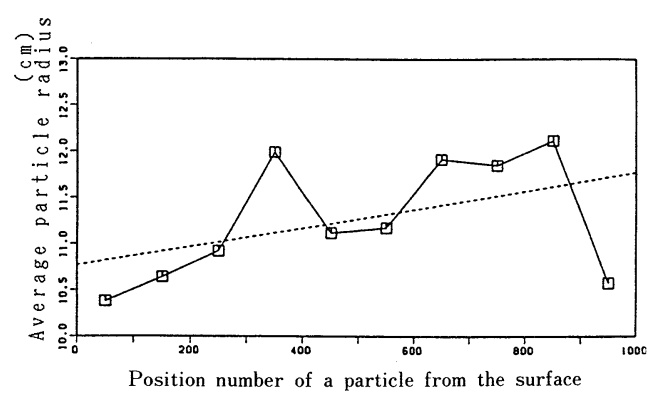

Fig. 9 Initial particle radius distribution in the cross section assembly of a dry avalanche.

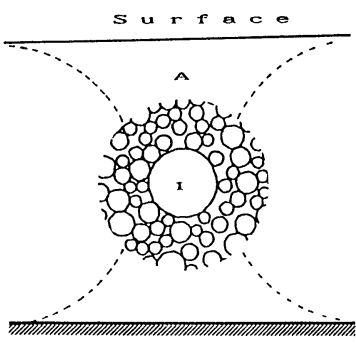

$B \circ t t \circ m$

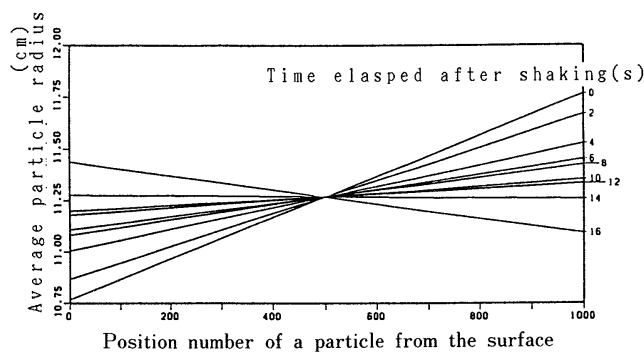

Fig. 10 Change in particle radius distribution with duration of shaking.

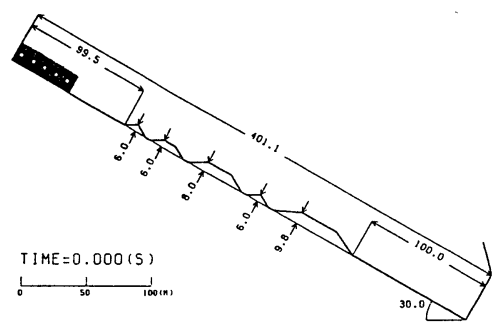

Fig. 12 Rough slope (Model slope 1) [Unit: Length (m), Angle (Degree)].

Fig. 11 Scheme for the upward flotation of a large particle.

particles (16 seconds) is shown in Fig. 8.

The initial particle-radius distribution is given in Fig. 10. The procedure used to construct this figure was as follows: The number of particles in Fig. 4 is 1000 . We gave every 1000 elements a sequence number in the order of the decreasing $z$-coordinate value (Fig. 4 ; from upper to lower particles). The average radii were calculated for every 100 particles in increasing order (1 through 100, 101 through 200, etc.) and were plotted on the vertical axis versus the average numbers on the horizontal axis (Fig.9). Therefore, the leftmost plot is the average radius for the uppermost to the 100 th particle. The dotted line in the figure is the regression line obtained by the least-squares method; the increasing tendency toward the right implies that the smaller the particles are the highest in the assembly and that they are concentrated. This tendency also is seen in Fig. 9 ; therefore, in the initial state there are more small particles concentrated in the high part of the assembly. We calculated the radius distributions and their regression lines (as above) every 2 seconds from 0 to 16 seconds (Fig. 10).

The numerals to the right of each line are times for analysis. These times increase in order from top to bottom; $0,2,4, \cdots, 16$ seconds. This implies that while being shaken, inverse grading took place in the vessel, which moved the larger particles upward and the smaller ones downward.

\section{( 3 ) Considerations}

In the cross-section model we have seen an inverse grading phenomenon that shifted the larger particles (larger boulders) upward and the smaller ones downward. The reason for this upward flotation of the large boulders is considered to be as follows: In order to move the large block I upward (Fig. 11), as much resistance is needed as to bring all the particles in domain A upward; but to move block I downward requires much more resistance, because the assembly is underlain by a rigid bottom. All in all, resistance will be less during upward movement than during downward movement. As a result, particles move toward the lesser resistance (upward), while being subjected to collisions and the external forces. The shallower the particles in the assembly, the less resistance is exerted from above, and flotation upward is easy. When a large particle is already on the surface, it will receive upward resistance exclusively and, its weight being supported from below, it does not subside. This reasoning applies only to large particles ; 


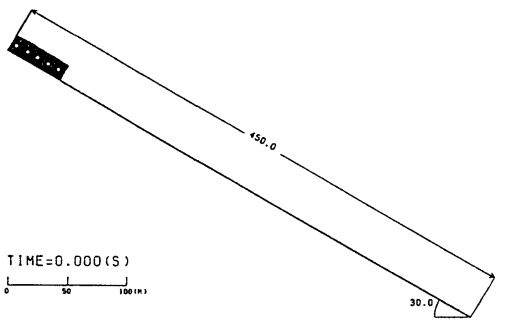

Fig. 13 Smooth slope (Model slope 2) [Unit: Length(m), Angle (Degree)].

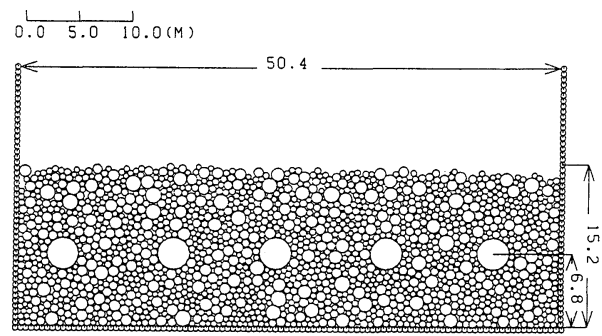

Fig. 14 Granular assembly model after compaction [Length (m)].
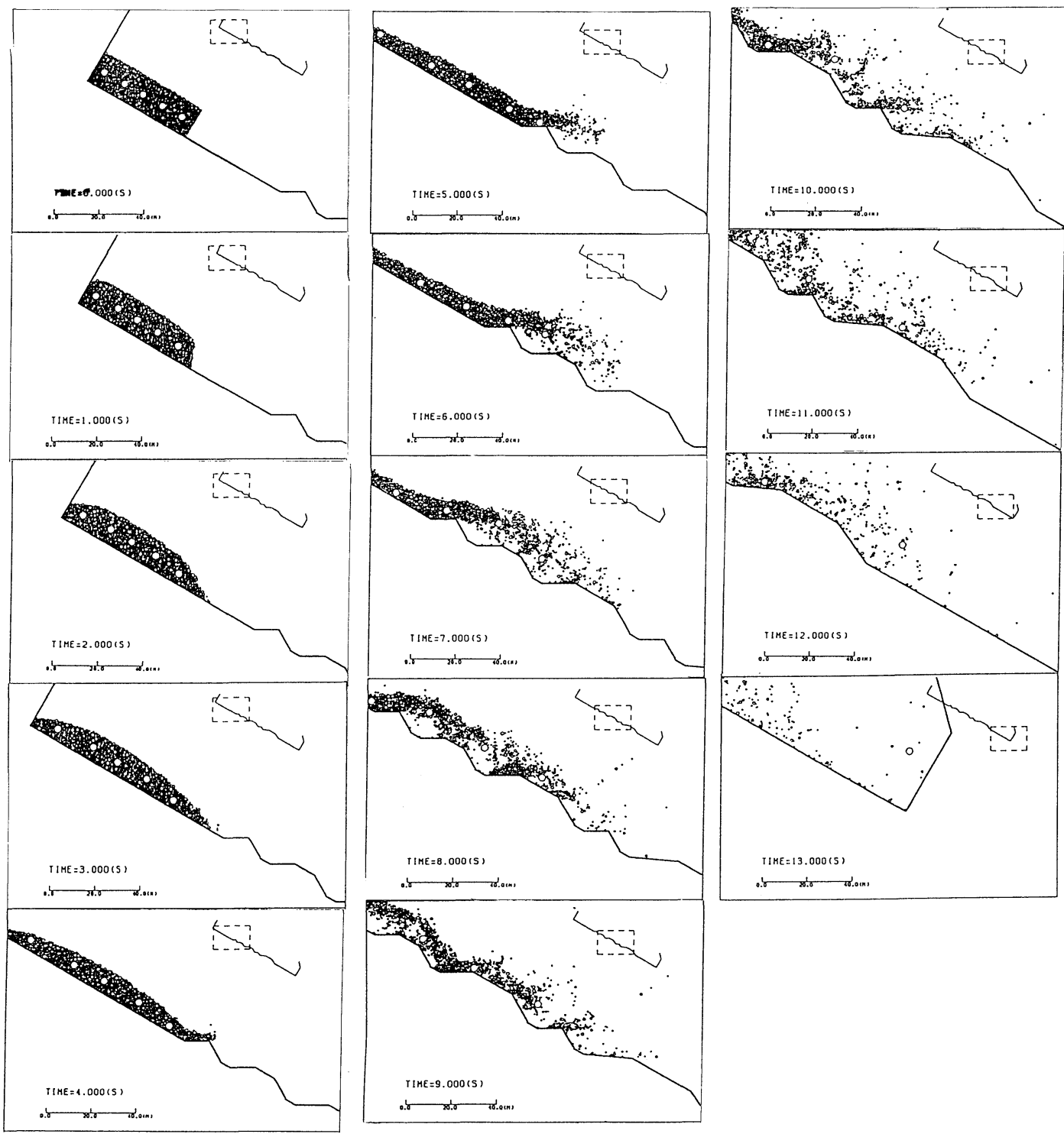

Fig. 15 Closeup of the dry avalanche simulated for a compacted assembly and rough slope. 
Table 2 Particles assembly data.

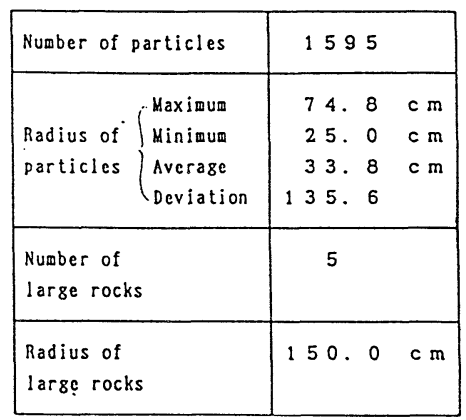

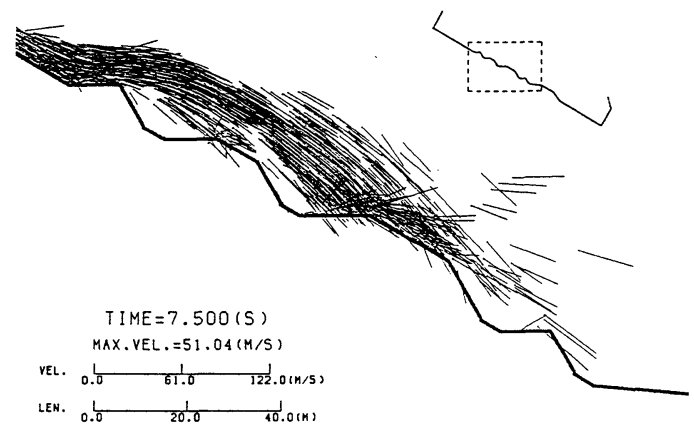

Fig. 17 Velocity distribution $7.5 \mathrm{sec}$ after the sliding of the compacted dry assembly.

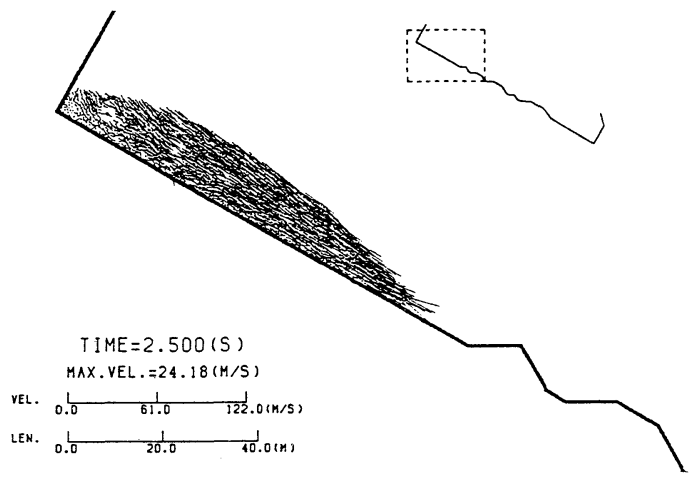

Fig. 16 Velocity distribution $2.5 \mathrm{sec}$ after the sliding of the compacted dry assembly.

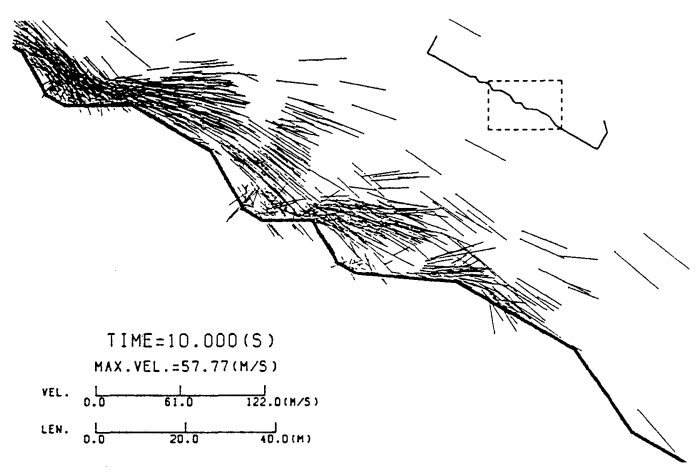

Fig. 18 Velocity distribution $10.0 \mathrm{sec}$ after the sliding of the compacted dry assembly.

small particles can subside downward through the pores in the assembly.

\section{ANALYSIS OF A ROCK AVALANCHE THAT FLOWS DOWN A SLOPE}

\section{(1) Explanation of the slope model}

Two kinds of slopes were prepared to simulate the flow phenomenon of a rock avalanche; Model slope 1 in Fig. 12 and Model slope 2 in Fig. 13. Model slope 1 has a monocline of ca. $100 \mathrm{~m}$ in the highest part, a randomly uneven part of ca. $200 \mathrm{~m}$ in middle, and again a monocline of ca. $100 \mathrm{~m}$ in the lowest portion, for a total length of ca. $400 \mathrm{~m}$. The number of the wall elements is 1046 . Model slope 2 is a monocline over its entire length of ca. $450 \mathrm{~m}$. The number of the wall elements is 979 . For both models the inclination of the monoclinic part of the slope is 30 degrees, and circular elements of radius of $25 \mathrm{~cm}$ are used.

\section{(2) Explanation of the particle model}

In the particle model, the radii of the particles range from a maximum of $74.8 \mathrm{~cm}$ a minimum of $25.0 \mathrm{~cm}$ with a mean of $33.8 \mathrm{~cm}$ and a variance of 135.6 (Table 2). The number of elements is 1595 . To observe the behavior of large boulders, we placed five elements each with a radius $150 \mathrm{~cm}$, at the depth of 10 meters then consolidated the particle assembly to a stable state under gravity. The final height was $15.2 \mathrm{~m}$, which corresponds roughly to a porosity of $16.3 \%$ (Fig. 14).

(3) Analysis of a rock avalanche that flows down an uneven model slope

The results shown in Fig. 15 are for a natural flow along an uneven slope under gravity. The front of the flow is shown enlarged in Fig. 15. The velocity distributions at 2.5, 7.5, 10 and 13 seconds are given in Figs. 16 through 19. The peak velocity is fairly high and particles that have once bounced up roll down the slope surface and are smashed then bounced back again in the air. From 10 to 13 seconds (Fig. 15) the large 

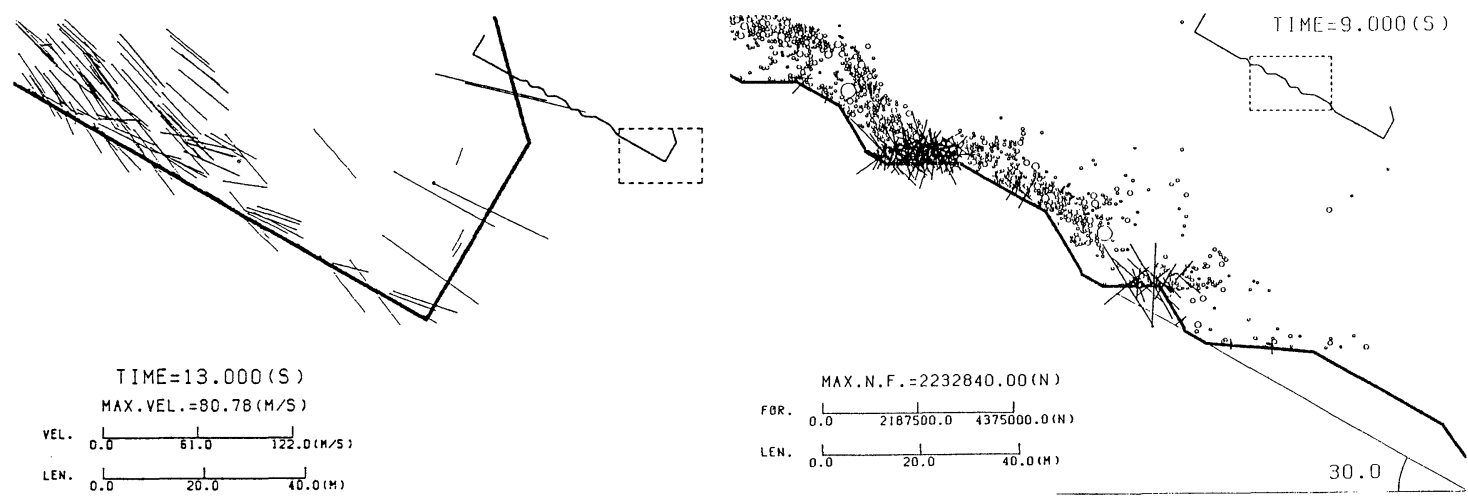

Fig. 20 Normal force distribution $9.0 \mathrm{sec}$ after the sliding

Fig. 19 Velocity distribution $13.0 \mathrm{sec}$ after the sliding of the compacted dry assembly. of the compacted dry avalanche.
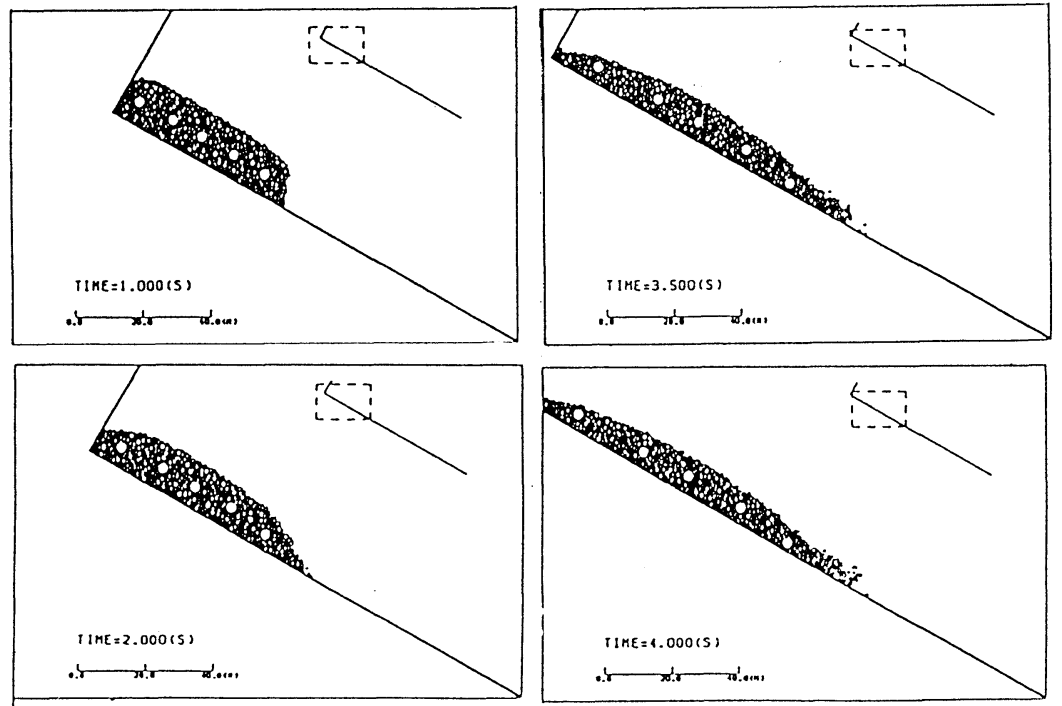

Fig. 21 Close-up of the dry avalanche simulated for a compacted assembly and even slope.

boulders (radius $75 \mathrm{~cm}$ ) are concentrated at the front part of the flow, which is characteristic of rock avalanches.

In the 1970 Peruvian earthquake a huge rock avalanche occurred on Mt. Huascaran, and sparks of light were observed. These sparks are presumed to have been produced by the collision of rock pieces in the avalanche. These sparks are considered to be simulated by collision forces between rock pieces, mainly those in the normal direction. The normal forces between particles are shown in Fig. 20.

According to this figure sparks are densely generated in the left half of the figure where particles that have been in the air collide again with the slope surface.

\section{(4) Analysis of a rock avalanche that flows down a smooth slope}

The behavior of rock particles that flows down an even slope was observed. The front part of the flow is shown enlarged in Fig. 21. In the first half of the period of analysis, the central part of the flow bulges in comparison to the front and back parts, but the difference in height becomes reduced gradually with time, resulting in nearly the same height over the total length at 6 seconds or later. The velocity distribution at 3. 5 seconds is shown in Fig. 22 and at 7. 0 seconds in Fig. 23. A comparison of the figures for 3.5 and 7.0 seconds shows the former is more regular than the later which indicates the disturbing effect of flow velocity because the average velocity must be lower at 3.5 seconds than at 7.0 seconds. Obviously there 


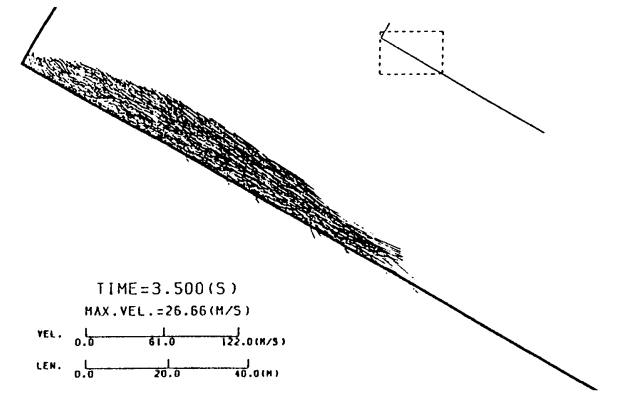

Fig. 22 Velocity distribution $3.5 \mathrm{sec}$ after the sliding of the compacted dry avalanche.

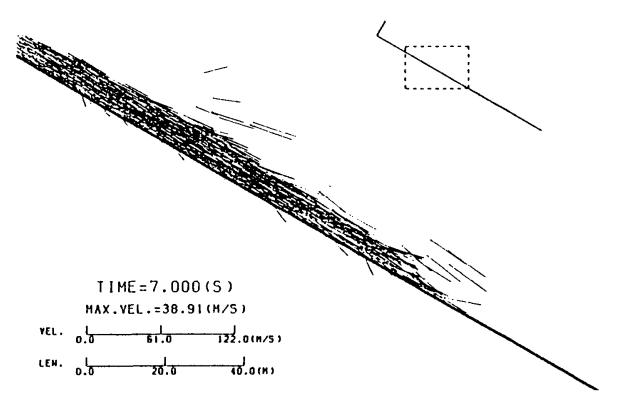

Fig. 23 Velocity distribution $7.0 \mathrm{sec}$ after the sliding of the compacted dry avalanche.

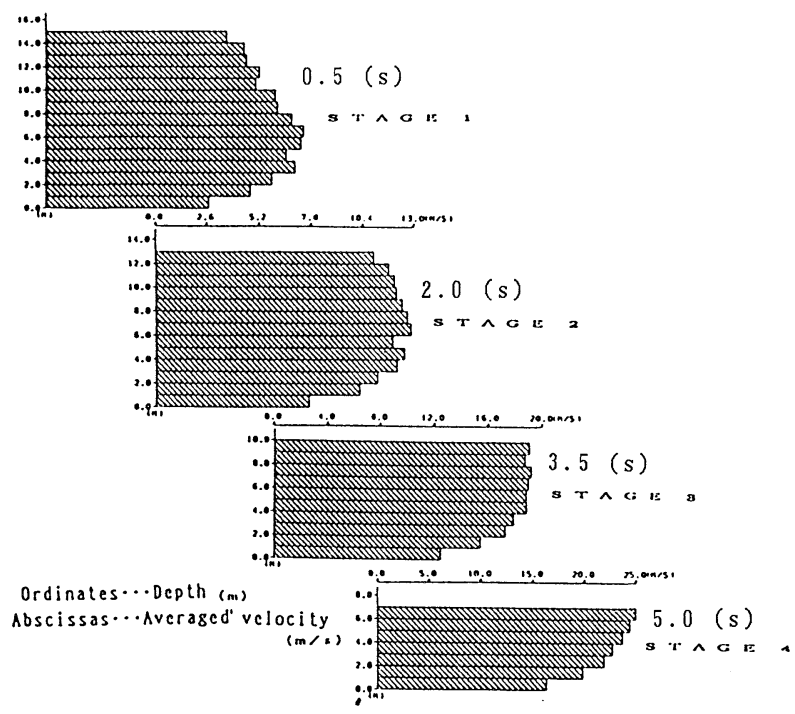

Fig. 24 Flow velocity at various depths for a compacted dry avalanche.

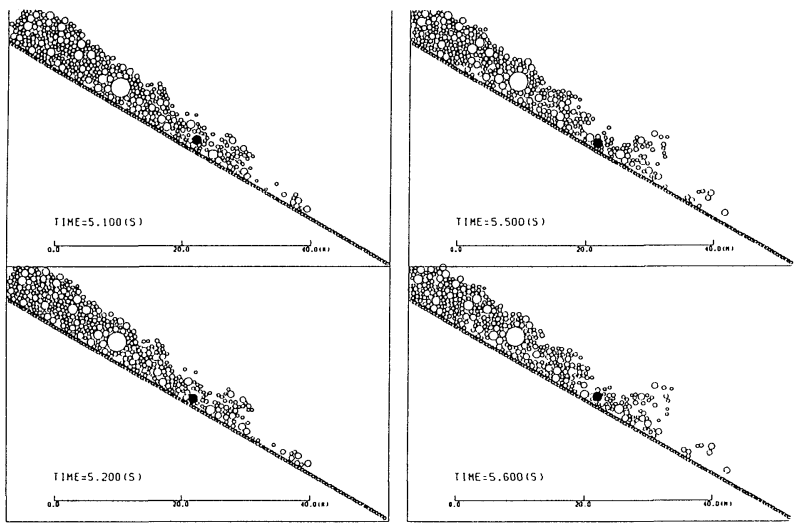

Fig. 25 Rolling phenomenon at the front of the flow.

are more chances for particles to be bounced out of the assembly in a rapid flow as greater forces are exerted on particles when they collide during rapid flow. Flow velocity distributions with depth at 0.5 , 2. 0, 3.5, and 5.0 seconds are shown in Fig. 24. The front part of the avalanche shows the phenomenon of the foremost rock pieces being rolled back under the avalanche as the front proceeds, as, also seen when the tracks of a bulldozer move forward. This phenomenon was first recognized by projecting a series of 


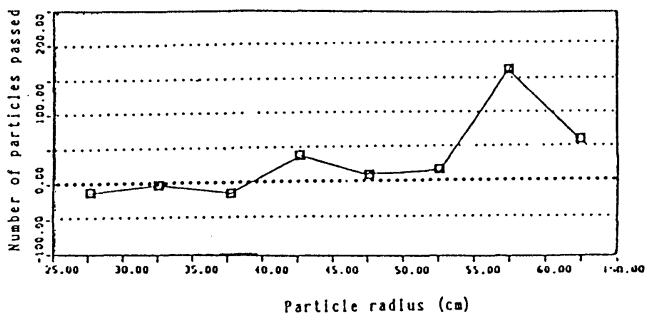

Fig. 26 How many particles does a given pass?

pictures (such as Fig. 25) through successively as movie on the CRT of a personal computer. Some of the element have here been colored black to show their position every 0.1 second.

\section{( 5 ) Considerations}

Concerning the mechanism for the concentration of large boulders at the front of the flow, Fig. 26 shows how many ranks (counted from the front backward) a class of particles of a certain radius range (e.g. 25 to $30 \mathrm{~cm}$ ) proceeded from its initial rank during the period of analysis. The longitudinal axis gives the rank and the horizontal axis the representative radius; the larger the radius is the greater the rank gained. Figs. 27 and 28 show the average radii of every 100 elements from the front to the rear of the flow for two stages; at the start and the end of analysis. The dotted lines in the figures are regression curves determined by the least squares method. When the line increases to the right, it indicates that particles are finer toward the front. In the initial stage in Fig. 27 the line increases to the right but with fairly large fluctuations. These fluctuations are natural when the initial radii and positions given randomly are taken into account. The regression line in Fig. 28 decreases to the right showing that particles are larger toward the front.

The mechanism for the concentration of large boulders at the front of the flow is considered to be as follows : The floating up tendency of large boulders is confirmed in the cross-section simulation in section 3 , and these large boulders will flow over the surface ; and, as the consequence of the greater flow velocity on the surface than in the bottom of the flow, the boulders will be transported toward the front and be concentrated there. These boulders at the forefront of the flow may be rolled into the bottom (Fig. 25), but the flotation process is repeated and they soon appear on the surface and are transported again to the front.

The rock avalanche is a flow phenomenon that has little viscosity and is accompanied by friction and particle collisions. Of the trial simulations of this phenomenon made by the DEM, the study reported here demonstrates its applicability. There are problems yet to be studied, however, in order to develop a model that incorporates the effect of water as in mud flows, and the effect of the shape of particles, must be taken into account because actual rock pieces are jagged not spheres.

\section{REFERENCES}

1) Mogami, T. : A Statistical Approach to the Mechanics of Granular Materials, Soils and Foundations, Vol.5, No.2, pp. 26-36, 1965.

2) Satake, M. : On Distortion of Tensor and Yield Criteria of Granular Materials, Int. J. Engineering Science, Vol. 19, No. 12. pp. 1643-1650, 1981.

3) Cundall, P. A. : A Computer Model for Simulating Progressive, Large Scale Movement in Blocky Rock Systems, Symp. ISRM, Nancy, France, Proc.2, pp. 129-136, 1971. 
4) Hakuno, M. and Hirao, T. : A Trial Related to Random Packing of Particle Assemblies, Proc. Japan Society of Civil Engineers, No. 219, pp. 53-63, 1973 (In Japanese).

5) Kiyama, H. and Fujimura, H. : Analysis on Gravity Flow of Rock Granular Assemblies by Cundall Model, Proc. Japan Society of Civil Engineers, No. 333, pp. 137-146, 1983 (In Japanese).

6) Kawasaki, R., Kiyama, H. and Fujimura, H. : DEM Analysis using Ellipsoidal Elements, Proc. Annual Meeting of Japan Society of Civil Engineers, 1986 (In Japanese).

7) Uemura, D. and Hakuno, M. : Granular Assembly Simulation with Cundall's Model for the Dynamic Collapse of the Structural Foundation, Structural Eng. /Earthquake Eng., Vol. 4, No.4. pp. 155 s-164 s, Proc. Japan Society of Civil Engineers, 1987.

8) Casaverde, L., Iwashita, K., Tarumi, Y. and Hakuno, M. : Distinct Element Method for Rock Avalanche, Structural Eng. /Earthquake Eng., Vol., 6, No.1, pp. 131 s-140 s, Proc. Japan Society of Civil Engineers, 1989.

9) Hakuno, M. and Tarumi, Y. : A Granular Assembly Simulation for Seismic Liquefaction of Sand, Structural Eng. /Earthquake Eng., Vol. 5, No. 2, pp. 333 s-342 s, Proc. Japan Society of Civil Engineers, 1988.

10) Iwashita, K. and Hakuno, M. : Modified Distinct Element Method Simulation of Dynamic Cliff Collapse, Structural Eng. /Earthquake Eng., Vol. 7, No.1, pp. 133 s-142 s, Proc. Japan Society of Civil Engineers, 1990.

11) Meguro, K. and Hakuno, M. : Fracture Analyses of Concrete Structures by the Modified Distinct Element Method, Structural Eng. /Earthquake Eng., Vol. 6, No.2, pp. 283 s-294 s, Proc. Japan Society of Civil Engineers, 1989.

(Received June 8 1989) 